Les sources de l'Histoire des Mines : Nouveaux outils, Nouvelles approches

\title{
«Impressions populaires »La collection de livrets conservée au MuCEM et la diffusion des savoirs par les livres de colportage (XVII ${ }^{\mathrm{e}}-\mathrm{XIX}^{\mathrm{e}}$ siècles)
}

Geneviève Deblock

\section{OpenEdition}

\section{Journals}

Édition électronique

URL : http://journals.openedition.org/dht/677

DOI : $10.4000 /$ dht. 677

ISSN : 1775-4194

Éditeur :

Centre d'histoire des techniques et de l'environnement du Cnam (CDHTE-Cnam), Société des élèves du CDHTE-Cnam

\section{Édition imprimée}

Date de publication : 1 décembre 2008

Pagination : 101-107

ISBN : 978-2-95-30779-2-6

ISSN : 0417-8726

Référence électronique

Geneviève Deblock, « «Impressions populaires »La collection de livrets conservée au MuCEM et la diffusion des savoirs par les livres de colportage (XVIIe-XIXe siècles) », Documents pour l'histoire des techniques [En ligne], 16 | $2^{\mathrm{e}}$ semestre 2008, mis en ligne le 04 octobre 2010, consulté le 08 septembre 2020. URL : http://journals.openedition.org/dht/677 ; DOI : https://doi.org/10.4000/dht.677 


\section{"Impressions populaires " \\ La collection de livrets conservée au \\ MuCEM ef la diffusion des savoirs \\ par les livres de colportage \\ (XVII ${ }^{\mathrm{e}} \mathrm{XIX}^{\mathrm{e}}$ siècles)}

Geneviève Deblock

MUCEM, CDHTE-Cnam

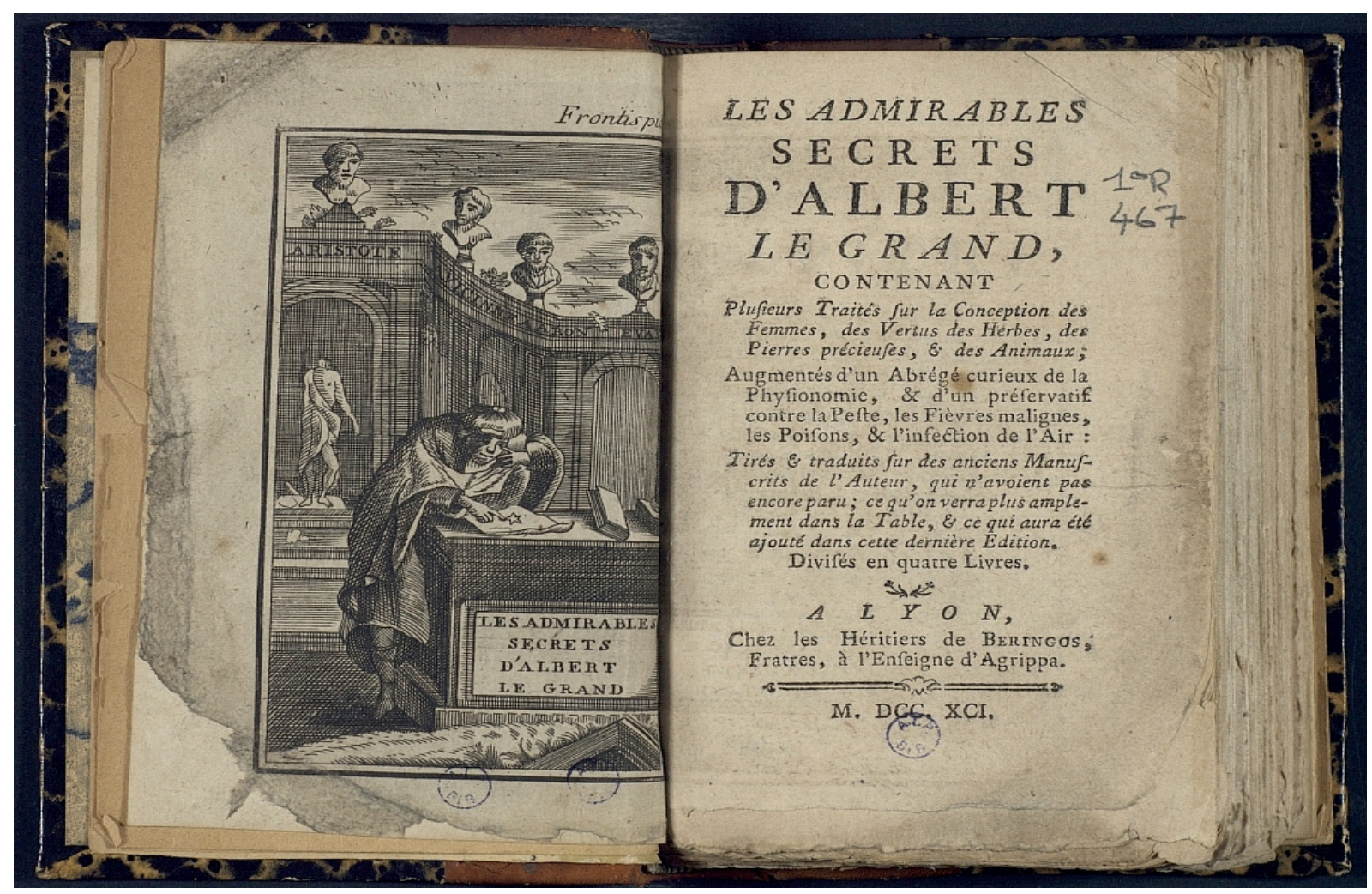

Les Admirables secrets d'Albert le Grand. A Lyon, chez les Héritiers de Béringos, Fratres, 1791

e MuCEM' conserve, avec la Bibliothèque natioLnale de France et la Médiathèque de Troyes, l'une des trois grandes collections françaises de littérature de colportage, un fonds exceptionnel par sa taille, par la diversité de ses titres et de leurs origines géographiques (plus de cent cinquante lieux de production y sont représentés). La Bibliothèque du MuCEM s'apprête à mettre en ligne cette collection dite d'« impressions populaires». Les ouvrages rassemblés

1 MuCEM, Musée des civilisations de l'Europe et de la Méditerranée sous ce terme générique sont presque entièrement des livres de colportage (XVII ${ }^{-} \mathrm{XIX}^{\mathrm{e}}$ siècles), auxquels ont été ajoutés cent cinquante ouvrages pour enfants, pour la plupart édités chez Pellerin à Épinal au $\mathrm{XIX}$ siècle, une cinquantaine de documents officiels (rapports, actes administratifs) émanant principalement de la Convention, une centaine de livres de colportage non français (allemands, anglais, espagnols, catalans, portugais, italiens, flamands, suisses, brésiliens), des textes en langues régionales et des livres qui ne sont pas de colportage, mais destinés aussi à un large public. Sur 3100 titres conservés, 2800 ont été numérisées (intégralement pour les deux tiers, la page de titre et la couverture pour le dernier tiers). Une deuxième campagne de numérisation doit être 
programmée. Elle complètera celle-ci et sera destinée à la collection de 1000 almanachs anciens. La médiathèque de Troyes a déjà mis en ligne les livres de la Bibliothèque bleve conservés dans ses murs. Un catalogue partagé des trois collections (non exhaustif) est consultable sur son site. II renverra à terme vers les sites des bibliothèques numériques. L'adresse est la suivante : "Librairie de colportage ", "Catalogue collectif de la Bibliothèque Bleve "):

http://www.mediathequeagglotroyes.fr/bmtroyes/num/fscatPRID.asp?PR=CATALOGUECOLLECTIONMULTI_O.

La Bibliothèque Bleve, ou plus généralement la littérature de colportage, est un mode d'édition et de diffusion. Les études d'histoire du livre et des pratiques culturelles ont permis de bien la connaître ${ }^{2}$. Elle fut initiée en France par Nicolas Oudot, imprimeur dans la ville de Troyes, en Champagne, entre 1600 et 1636. Des formes très semblables à celle-ci existent dans d'autres pays d'Europe (chapbooks anglais ${ }^{3}$, littérature de cordel en Espagne ${ }^{4}$ ) ; le genre n'est pas exclusif à l'Europe, une étude en a identifié aussi au Brésil ${ }^{5}$. II s' agit de petits livres médiocrement imprimés

2 Alfred Morin, Catalogue descriptif de la bibliothèque bleve de Troyes (almanachs exclus), Genève, Droz, 1974 ; Robert Mandrou, La culture populaire aux $17^{\mathrm{e}}$ et $18^{\mathrm{e}}$ siècles, Paris, Imago, 1985 ; Geneviève Bollème, La Bibliotheque bleve. La littérature populaire en France du XVle au XIXe siècle, Paris, Julliard, collection Archives, 1971 ; Margaret Spufford, The great reclothing of rural England. Petty chapmen and their wares in the seventeenth century, Londres, Hambledon Press, 1984 ; Laurence Fontaine, Histoire du colportage en Europe, XVe-XIXe siècle, Paris, Albin, Michel, 1993 ; Lise Andries, Geneviève Bollème, La Bibliothèque bleve. Literature de colportage, Robert Laffont, collection Bouquins, 2003 ; William Eamon, Science and the secrets of nature. Books of secrets in medieval and early modern culture. Princeton, Princeton University Press, 1994.

3 M. Spufford, The great reclothing of rural England, op. cit. ; id., Small books and pleasant histories, Cambridge, Cambridge University Press, 1985.

4 Jean-François Botrel, « Une bibliothèque bleue espagnole? Les historias de cordel (XVII ${ }^{-}-\mathrm{XX}$ siècle) $\|$, et particulièrement la note $n^{\circ} 69$ « livres utiles comme...Secretos de utilidas y recreo, La cocinera catalana,... "), dans Thierry Delcourt et Élisabeth Parinet éd., La Bibliothèque bleve et les littératures de colportage, actes du colloque organisé par la bibliothèque municipale de Troyes, en collaboration avec l'École nationale des Chartes (Troyes, 12-13 novembre 1999), Paris, École des Chartes/Troyes, La Maison du Boulanger, 2000, pp. 193-209.

5 Idelette Musart-Fonseca, " L'héroïsme féminin dans la littérature de cordel brésilienne ॥, dans Th. Delcourt, Élisabeth Parinet éd., La Bibliothèque bleve et les littératures de colportage, op. cit., pp. 221-233 ; Marie-Dominique Leclerc, « Bi- sur feuilles de mauvaise qualité, protégés par une couverture en papier grossier souvent bleue, qui sont édités en grand nombre ${ }^{6}$. Ils sont ensuite vendus par des colporteurs à des prix cinq à dix fois moins élevés que les prix habituels en librairie. Cette formule astucieuse d'édition et de diffusion persiste en France durant presque trois siècles, jusqu'aux années 1860. Très rentable pour les libraires qui la pratiquent, elle favorise une large diffusion, dans les villes puis aussi dans les campagnes. Le nombre des titres courants de la Bibliothèque bleve n'est pas très important. Selon Geneviève Bollème, il serait de quatre cent cinquante environ. Si l'on compte les rééditions et les ouvrages qui n'ont pas eu une grande durée de vie, la production entre le XVIle et le milieu du XIXe siècle pourrait s'élever à cinq mille titres ${ }^{7}$.

La plupart des ces petits livres de poche mal reliés et longtemps méprisés a disparu, et ils ne figurent pas dans les inventaires notariés de successions. Cependant, les ex libris et les notes manuscrites figurant sur les pages de garde apportent des informations très précieuses ${ }^{8}$. Ils donnent des indications sur les usages de ces publications et leurs lecteurs, ainsi que sur les collectionneurs qui les ont rassemblées plus tard. L'exemple du fonds d' "Impressions populaires ॥ est intéressant à ce sujet, car il révèle une volonté de sauvegarder, de rassembler et de faire figurer dans une collection muséale ces textes alors oubliés de tous et longtemps mal étudiés. La collection est inaugurée par le don d'une édition du Maréchal expert ${ }^{9}$, au tout nouveau Musée national des arts et traditions populaires, en 1938, par son premier directeur Geor-

bliothèque bleue et littérature de cordel $»$, Le vieux papier, avril 2007, pp. 49-60.

6 II est difficile de donner une idée exacte des tirages effectués. Leur importance évolve d'ailleurs au cours des années. Mais il est certain que les livres de colportage ont fait la fortune des imprimeurs-libraires. L'inventaire après décès de Jacques II Oudot en 1722 le démontre. Voir Robert Mandrou, La culture populaire aux 17e et $18^{e}$ siècles, op. cit., pp. 39-40 et 199-201.

7 Geneviève Bollème, La Bible bleve, anthologie d'une littérature populaire. Paris, Flammarion, 1975, p. 18.

8 Marie-Dominique Leclerc, "Ex libris manuscrits et notes dans les impressions de grande diffusion (XVII $-\mathrm{XIX}^{\mathrm{e}}$ siècle) ", Histoire et civilisation du livre, vol. 2, Lyon et les livres, Genève, Droz, 2006, pp. 323-345.

9 Le maréchal expert, traitant du naturel et des marques des beaux et bons chevaux, de leurs maladies, et remèdes d'icelles. Avec un examen en forme de l'état du maréchal ; et une description de toutes les parties et ossemens du cheval. Augmentée d'une seconde partie, contenant plusieurs recettes approuvées du sieur de L'Epinet, par feu N. Beaugrand, M. maréchal à Paris, chez Nipolahc, imprimeur [Caen, Chalopin], sd [1746-1786] (cote 1R 2). 
ges-Henri Rivière, qui écrit quelques années plus tard : " ainsi, la littérature de colportage, qu'elle donne ou qu'elle reçoive, participe-t-elle aux grands courants de civilisation. Outre l'histoire littéraire, [elle] intéresse de vastes secteurs de l'histoire sociale ${ }^{10}$. De nouvelles acquisitions entrent au Musée en 1941 avec le don de huit livrets de colportage par le libraire Jean Deschamps. Suit alors une série d'achats s'échelonnant de 1948 à 1989, parmi lesquels sont représentées d'importantes collections particulières (collection Bureau, collection Flocon ayant appartenu à Georges Heilbrun, collection du docteur René Hélot ${ }^{11}$, chez Gérard Oberlé, collection Louis Ferrand) ${ }^{12}$.

Comment décrire ces ouvrages, souvent sans mention d'auteur ni de date d'édition? Leurs contenus sont extrêmement divers : des textes datant du Moyen Âge et de la Renaissance tombés dans le domaine public, textes religieux pour une très grande part, romans de chevalerie, calendriers, livres pratiques d'apprentissage, livres de secrets et de médecine, magie et sciences occultes. À cela, s'ajoutent au fil du temps, de nombreux contes au XVIII ${ }^{e}$ siècle, des abécédaires, livres d'enseignement, livres de civilité, des textes satyriques concernant la vie quotidienne et les métiers, burlesques et en langages poissards, des jeux, chansons, saynètes, récits de faits divers, ainsi que quelques ouvrages scientifiques et techniques, quelques textes d'actualité plus nombreux dans les almanachs. Certains titres ont plusieurs rééditions, avec ou sans variantes ou actualisations au fur et à mesure du temps. Ils sont en général de petite taille (10 à $20 \mathrm{~cm}$ ), et peu épais. Leur mise en page est serrée, à l'économie. Leur iconographie est pauvre, et constituée de bois souvent usés. Leur structure très simple est faite de paragraphes brefs, et ils sont souvent dépourvus de table des matières.

Vouloir répertorier dans cet ensemble hétérogène d'« impressions populaires », les ouvrages divers relatifs aux savoirs pratiques, aux apprentissages ou plus précisément aux techniques, est une entreprise téméraire. D'une part, leurs contenus sont souvent hétérogènes, ou encyclopédiques. D'autre part, la majorité des livres de colportage se réfère à un système d'interprétation du monde bien différent du nôtre dans lequel,

10 Pierre Brochon, Le livre de colportage en France depuis le XVle siècle, sa littérature, ses lecteurs, préface de Georges Henri Rivière, Paris, Gründ, 1954.

11 René Hélot, La Bibliothèque bleve en Normandie, Roven, Société rovennaise des bibliophiles, 1928.

12 Monique Lambert, « La collection de 3000 livrets bleus de la Bibliothèque des arts et traditions populaires ॥, Bulletin d'information de l'ABF, no 146, ler trimestre 1990, pp. 6-20. comme le fait remarquer Lise Andries, « il n'existe pas alors de frontière entre la technique et la magie $\|^{13}$, un thème familier des anthropologues mais moins des historiens ${ }^{14}$. Leur lecture fournit des informations sur les connaissances scientifiques et techniques qui circulaient via l'imprimé auprès des populations des villes et des campagnes entre le XVII et le XIXe siècle. Du moins est-il possible de dénombrer environ deux cent cinquante titres se rapportant aux savoirs, aux sciences et aux techniques, mais il faudrait y ajouter le corpus déjà signalé d'abécédaires, de livres d'enseignement, de livres de jeux, de livres de civilité, d'ouvrages entièrement consacrés à la magie et la divination qui participent tous de l'univers des arts et de l'acquisition de gestes et de méthodes, sous l'Ancien Régime.

Le Grand calendrier des bergers ${ }^{15}$ est un titre emblématique de la Bibliothèque bleve. Issu d'un ouvrage édité à Paris en 1491 chez Guy Marchand, et composé lui-même de divers textes plus anciens, il est le modèle des almanachs qui ont eu un si grand succès par la suite. Son inspirateur, « un berger gardant des brebis aux champs n'étoit nullement éclairé, n'y avoit aucune connoissance des Écritures ; mais seulement par son grand sens naturel \& bon entendement, disoit : quoi que vivre et mourir dépend entièrement de Dieu, l'homme doit pourtant naturellement vivre jusqu'à LXXI ans ou plus ${ }^{16}{ }^{16}$. L'ouvrage est une compilation des savoirs utiles au quotidien : le calendrier et la connaissance du temps, les heures, les jours, la nuit, les fêtes religieuses, les saisons, le déroulement de la vie, les astres, les signes du zodiaque et la divination, la terre et les climats, l'anatomie du corps humain, les maladies et les remèdes pour les hommes et pour les animaux, la description des oiseaux qui nous côtoient. On se trouve ainsi à la frontière entre la transmission orale et la diffusion écrite.

Les livres de secrets, rassemblés en un seul volume (de 8 à 250 pages), et sans grande logique de classe-

13 Lise Andriès, Le grand livre des secrets. Le colportage en France aux $17^{\mathrm{e}}$ et $18^{\mathrm{e}}$ siècles, Paris, Imago, 1994.

14 Marcel Mauss, Esquisse d'une théorie générale de la magie (1902-1903), dans id., Sociologie et anthropologie (1950), Paris, Presses universitaires de France, Quadrige, 1995.

15 Le grand calendrier et compost des bergers. Composé par le Berger de la grand montagne, avec le compost naturel reformé selon le retranchement des dix jours, par le Pape Grégoire III. Ensemble la manière comme se doit gouverner le berger pour empêcher qu'aucuns sorciers ne fassent mourir leurs troupeaux, avec toutes choses nécessaires pour se régler en leur art, à Troyes, chez Jacques Oudot, s.d., permission de 1705 (cote 2R 4).

16 Le grand calendrier et compost des bergers, op. cit., «prologue de l'auteur qui a mis par écrit le calendrier des bergers »" 
ment, proposent eux aussi tout un savoir encyclopédique se rapportant essentiellement à des préoccupations domestiques ${ }^{17}$. À côté de procédés relevant de la magie et des sciences occultes, ils donnent des recettes utiles pour la vie quotidienne, telles que « rassembler le verre et le cristal rompus ", " faire du savon ", " faire un châssis qui semble de verre ", " pour faire que les punaises ne te puissent point nuire la nuit ", " pour coller tout ce qui te plaira ${ }^{1}{ }^{18}$, des compilations de secrets de métiers, ainsi que des recettes de médecine, de cuisine, de confection de cosmétiques, « pour faire eau rouge pour peindre le visage des femmes $\|{ }^{19}$. Comme l'explique Catherine Lanoë, cuisine et médecine relèvent de préoccupations très proches : « en utilisant souvent les mêmes ingrédients et les mêmes méthodes, en tissant maintes correspondances entre intérieur et extérieur du corps, ces trois techniques fondamentales et liées (se soigne, se nourrir, s'entretenir) permettent d'exercer un premier contrôle sur la vie de I'homme $\|{ }^{20}$. II s'agit aussi de se distraire, et l'on perçoit ici les liens entre les techniques, le jeu et le plaisir ${ }^{21}$ : il est ainsi proposé dans le Bastiment des receptes ${ }^{22}$, après « plusieurs receptes de grande vertu, lesquelles sont pour te donner grande vérité, maintenant par manière de passe-temps... je te veux écrire quelques petites choses plaisantes, desquelles tu pourras prendre plaisir et récréation », par exemple « pour faire qu'un chien ou un cheval te semblera tout vert». Le texte résonne avec les propos du « Berger de la grand montagne », exprimant le désir de vivre «longtemps, sainement et joyeusement ", non sans écho avec le souci de préservation de soi hérité de la médecine galénique ${ }^{23}$. L'humour et le divertissement sont étroitement liés à la transmission des savoirs pratiques dans la collection d'« impressions populaires ». « Nous avons... la prétention de plaire à ceux qui aiment à dépen-

17 L'homme et son corps dans la société traditionnelle, exposition, Paris, 1978/Nantes, 1978-1979 (commissaire Françoise Loux), Paris, RMN, 1978, pp. 117, 130, 131.

18 Le bastiment des receptes traduit de l'italien en françois. Et augmenté d'une infinité de beaux secrets depuis peu en usage. Avec un autre petit traité de recepte intitulé le grand jardin, Troyes, s.d. (cote 1R 500).

19 Le bastiment des receptes, op. cit.

20 Catherine Lanoë, La poudre et le fard. Une histoire des cosmétiques de la Renaissance aux Lumières, Seyssel, Champ Vallon, 2008, p. 342.

21 François Sigaut, "Les techniques dans la pensée narrative ॥, Techniques \& culture, 2004, vol. 43-44, pp. 191-213.

22 Le Bastiment des receptes, op. cit.

23 Catherine Lanoë, "Galien ou Paracelse, conserver ou transformer ? Les cosmétiques à la Renaissance 1 , Journal de la Renaissance, III, 2005, pp. 193-206. ser leurs loisirs à des distractions agréables et qui, dans des jeux en apparence frivoles, savent trouver une utile recette pour chasser l'ennui ou pour développer de plus en plus leur intelligence » est-il écrit dans un livre de Tours d'escamotage, de prestidigitation et d'adresse ${ }^{24}$. L'antique métis se coule dans la culture ludique de l'adresse et de la combinatoire qui accompagne l'essor des réductions en art, le goût des artifices et des curiosités, la montée des plaisirs optiques auprès de publics élargis ${ }^{25}$.

C'est dans ce contexte qui promeut les savoirs comme une « intelligence ${ }^{26}{ }^{26}$, comme l'exercice du génie (un « faire »), que se déploient l'arithmétique, l'économie, l'arpentage dans ces traités, plus particulièrement au XIXe siècle, avec les productions de François-Bertrand Barrême (1638-1703) ${ }^{27}$, ou bien dans de véritables petites encyclopédies qui réunissent en un seul volume de quelques pages, un savoir pratique utile au quotidien, et quelques données d'histoire et de géographie. On rencontre ainsi des titres extrêmement longs, rédigés certainement pour appeler le client, telle La Nouvelle science des gens de campagne, mise pour les calculs, en harmonie avec les nouvelles mesures, contenant la manière de dresser différents actes civils, un Abrégé de l'arpentage, un traité sur la culture des jardins et des arbres fruitiers, des remèdes éprouvés pour diverses maladies, un traité d'arithmétique décimale où l'on peut apprendre facilement, et même seul, à compter, chiffrer et calculer toutes sortes de sommes, et des modèles de lettres sur toutes sortes de sujets ${ }^{28}$, en 92 pages. La Description des principaux faits d'armes depuis 1792 jusqu'en $1815^{29}$, contient aussi sur un ensemble de 12 pages, des notions sur le commerce, l'arithmétique, la géographie, qui n'ont aucun rapport avec le titre

24 L'ancienne et la nouvelle collection des tours d'escamotage de prestidigitation et d'adresse...mis à la portée de tous, Paris, s.d. (cote 1R 506).

25 Pascal Dubourg-Glatigny, Hélène Vérin éd., Réduire en art. De la Renaissance aux Lumières Paris, MSH, à paraître ; Liliane Pérez, «Technology, curiosity and utility in France and in England in the XVIIIth century $"$, dans Bernadette Bensaude-Vincent, Christine Blondel éd., Sciences and Spectacle in European Enlightenment, Aldershot, Ashgate, 2008, p. 25-42. 26 Hélène Vérin, La gloire des ingénieurs. L'intelligence technique du XVI e au XVIII e siècle, Paris, Albin Michel, 1993.

27 Comptes faits de barème... Ouvrage utile et indispensable à tous les marchands et cultivateurs, Epinal, s.d., début du XIXe siècle (cote OIR 104).

28 La nouvelle science des gens de Campagne, mise pour les calculs..., Épinal, s.d., XIXe siècle (cote IR 366).

29 La description des principaux faits d'armes depuis 1792 jusqu'en 1815, Bernay, Impr. Duval, 1845 (cote 1R 1076) 
du livret, mais qui sont annoncées dans un sommaire dès la première page.

Dans la grande diversité des domaines traités, quelques métiers se détachent d'emblée, et font l'objet d'ouvrages spécialisés. Destinés à un public plus professionnel, ceux-ci ont souvent un auteur identifié, et sont en général plus épais et plus structurés. On sait l'importance qu'avait quotidiennement le maréchal dans les sociétés urbaines comme dans les sociétés rurales, où il apportait sa double compétence d'artisan du fer et de garant de la santé des chevaux ${ }^{30}$. Des ouvrages sont consacrés à ce métier, et à partir de la fin du XVIII siècle, auX arts vétérinaires ${ }^{31}$. II existe aussi des manuels de médecine et de santé ${ }^{32}$, auxquels on peut lier les manuels de cuisine ${ }^{33}$ et les manuels de

30 Daniel Roche, La culture équestre de l'Occident XVle-XIX siècle, Paris, Fayard, 2008, pp. 317-353.

31 Plusieurs éditions du Maréchal expert, de Nicolas Beaugrand ; L'art de panser et de guérir toutes les maladies des chevaux, s.l., s.d. (cotes IR 345 et IR 828) ; J.G. Boutrolle, Le parfait bouvier, ou instruction concernant la connoissance des boeufs et vaches, leur âge, maladies et symptômes, avec les remèdes les plus expérimentés, propres à les guérir. On y a joint deux petits traités pour les moutons et porcs.... Angers, 1784 ; Admirables secrets et remèdes pour guérir les boeufs, vaches.... du sieur Jean-Pierre Capt, Lyon, 1764 (cote 1R 858-2) ; Le trésor du laboureur, ou l'art de guérir les chevaux et bêttes à cornes. Ouvrage approuvé des écoles vétérinaires de Paris, Lyon, Roven etc., mis à la portée de tous les propriétaires de chevaux et bestiaux, et contenant des remèdes infaillibles, d'une exécution facile et peu dispendieuse, pour les maladies les plus graves et les plus fréquentes, par Jacques Richelet, artiste vétérinaire de Lyon, Epinal, s.d. (cote 1R 86).

32 Le trésor des pauvres, auquel sont contenus plusieurs remèdes, breuvages, oignements, emplastres, pillules, electuaires, préservatifs, et receptes côtre toute sorte de maladies, composé par maistre Arnoul de Ville-Nove, et autres docteurs en médecine, Lyon, 1590 (cote 1R 516) ; Le manuel médical de famille ou remèdes pour les maladies et accidents qui exigent de prompts secours, par Joseph-Xavier Tissot, médecin à Piolenc, Orange, 1815 (cote 1R 934) ; L.-J.-R. Gaux, Petit manuel de santé, d'utilité et d'agrément, ou recettes procédés et remèdes utiles et éprouvés, Paris, 1824 (cote 1R 692) ; Trésor de la propreté, de la santé et de la toilette, de A. et M. Plaisance, Lyon, 1836 (cote IR 1385).

33 Le cuisinier françois, enseignant la manière d'apprêter \& assaisonner toutes sortes de viandes grasses \& maigres, légumes \& pâtisseries en perfection, etc., de François-Pierre de La Varenne, s.d., XVIII siècle (cote IR 584) ; Le petit cuisinier familier, ou la manière d'apprêter les alimens agréablement et à peu de frais, Dole, chez F. Prudon, s.d. (cote OIR 48) ; Petite cuisinière habile ou l'art d'apprêter les alimens avec délicatesse et économie, par Madame FR, nouvelle édition corrigée et augmentée, Paris, chez Fridel et Gasc, 1822 jardiniers ${ }^{34}$. Le cuisinier français ${ }^{35}$, de François Pierre de La Varenne, dont la première édition date de 1651 (Paris, chez Pierre David), est un véritable succès de librairie. II est édité dès 1661 chez Nicolas II Oudot, à Troyes, après l'expiration du privilège daté de 1651 pour 10 ans, et fait l'objet de rééditions dans la Bibliothèque bleue jusqu'au XIXe siècle.

Enfin, la volonté d'offrir un livre abrégé, simplifié, accessible plus rapidement, plus aisément et à un moindre prix, se retrouve souvent, sous des titres quelquefois d'accès assez difficile. Le Calendrier du monde ${ }^{36}$, " supplément du Calendrier universe ${ }^{37}$ imprimé en 1731 chez Rollin... est destiné à tous les hommes sans exception ». " Pour trouver tout l'ordre des tems, il ne faut que ce petit volume, et n'avoir point d'autre science, que de scavoir bien lire \& se donner la peine de faire quelque attention en lisant ». " Ce traité ne sera pas fort long et on l'abrégera autant qu'il sera possible ", lit-on dans Les Admirables secrets d'Albert ${ }^{38}$. Nicolas Bonnefons, auteur du Jardinier français écrit « pour ceux qui n'y ont aucune connaissance, ou bien petite ${ }^{39}$; " pour instruire tant de pauvres gens... je me suis rendu le plus intelligible qu'il m'a été possible dans les termes les plus communs de notre langue », ajoute-t-il. Mais l'ouvrage est aussi destiné à tous, aux sommeliers « qui avoueront qu'il ne leur est pas entièrement inutile » aux « personnes de qualité ", ou aux « bourgeois qui ont des maisons de plaisir proches de Paris $»$.

En conclusion, cette collection rassemble des textes édités sur trois siècles sans grands changements semble-t-il, si ce n'est progressivement au XIXe siècle. Destinés à des publics différenciés, ils présentent surtout des savoirs pratiques, dépourvus de commentaires théoriques. Le ton est souvent familier. Les auteurs, ou compilateurs, tentent d'informer leurs lecteurs dans un langage simple et bref. Loin d'une histoire héroïque

(cote 1R 321).

34 Nicolas de Bonnefons, Le jardinier françois. Qui enseigne à cultiver les arbres et herbes potagères, avec la manière de conserver les fruits et faire toute sorte de confitures, conserve et massepain, dédié aux dames, Troyes, 1723 (cote 1R 1299) ; Le nouveau jardinier françois, revû, corrigé et augmenté de la vertu des herbes, Limoges, s.d. (cote IR 489).

35 Le cuisinier françois, textes présentés par Jean-Lovis Flandrin, Philip et Mary Hyman. Paris, Montalba, 1983.

36 Le calendrier du monde, où l'on donne une méthode très aisée de trouver à chaque année... tout l'ordre des tems.... Bruxelles, 1743 (cote 1R 229).

37 Calendrier universel, ou Almanach chronologique et perpétuel..., par le R. P. de Rebeque Soc. J., Paris, Rollin, 1731 38 1791. Cote 1R 467.

39 Op. cit. 


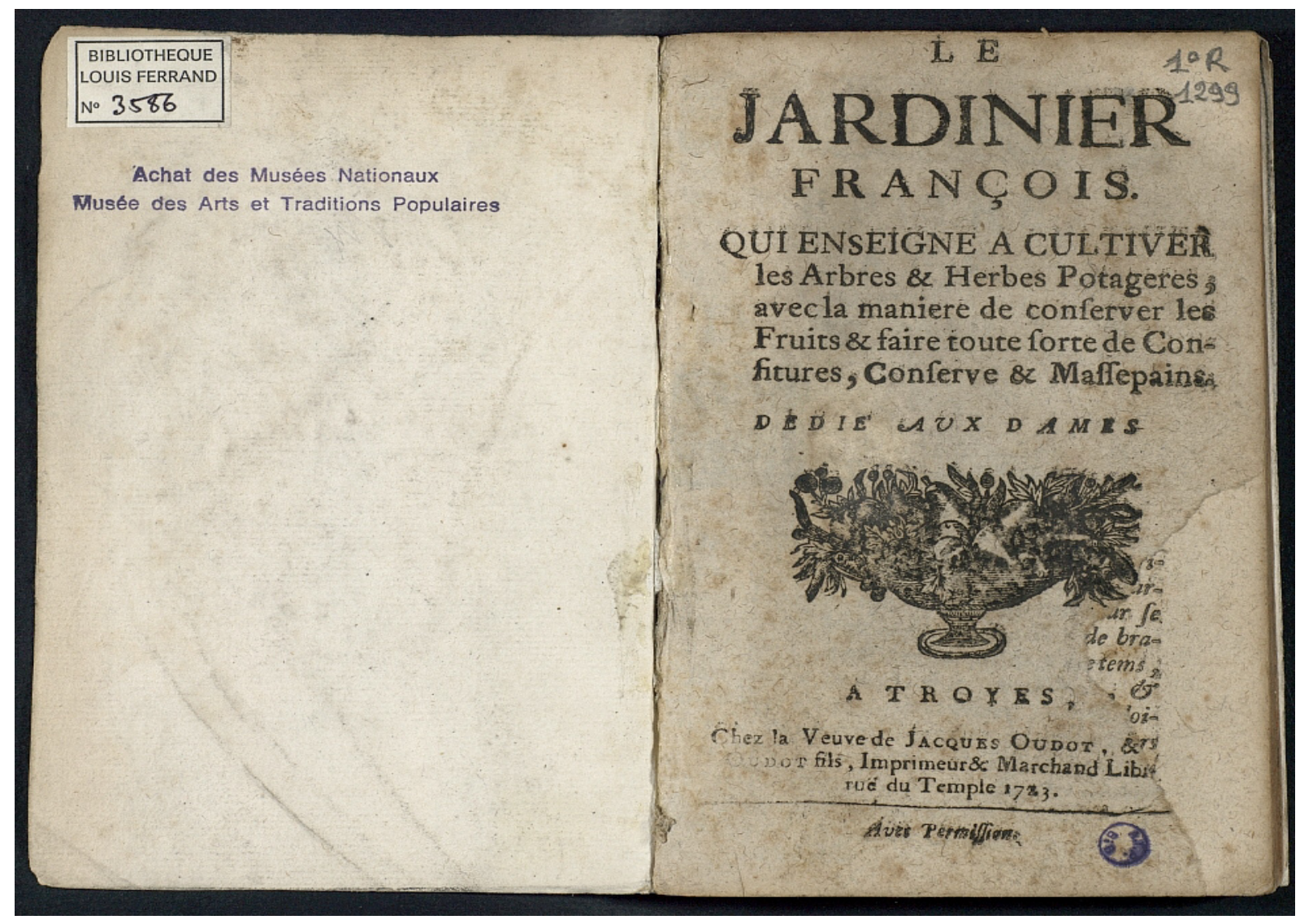

Le Jardinier françois. A Troyes, Chez la Veuve de Jacques Oudot et Oudot fils, 1723

et linéaire du progrès, cette littérature, à la croisée des livres de secrets (marqués par la magie et l'alchimie), des réductions en art et de la littérature d'usage (prospectus, modes d'emplois) ${ }^{40}$, révèle la complexité de

40 Liliane Hilaire-Pérez, Marie Thébaud-Sorger, « Les techniques dans l'espace public. Publicités des inventions et littérature d'usage en France et en Angleterre au XVIIIle siècle ", savoirs pratiques, en décalage avec le mouvement inventif tel qu'il est habituellement restitué dans les grands traités des sciences et des arts : une culture technique dans laquelle le geste, l'acte (matériel, intellectuel) participent de visions du monde qu'il s'agit d'identifier et de comprendre.

Revue de synthèse, 2006, n² 2, pp. 393-428. 


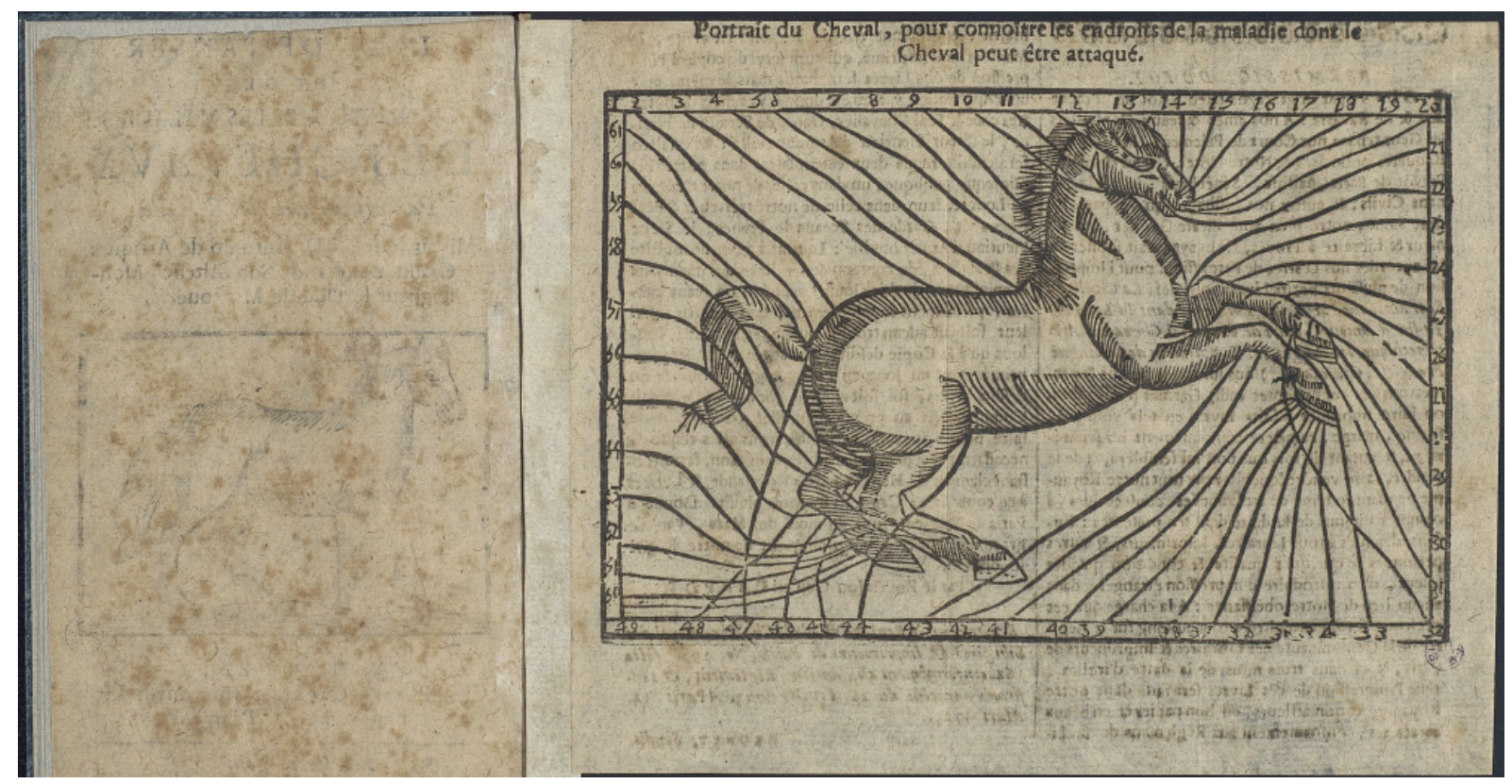

Portrait du cheval pour connaître les endroits de la maladie dont le cheval peut être attaqué [L'Art de panser et de guérir toutes les maladies des chevaux (cf note $n^{\circ} 18$ )]

\section{E M A R E H A L E X P $\quad$ E R $\quad T, 1^{\circ} R$ CONTENANT 1012 \\ PLUSIEURS RECETTES}

Approuvées du fieur DE L'EsPINEY, Gentilhomme Périgourdin, pour toutes les maladies $\&$ accidents qui arrivent aux Chevaux.

A VEC FI G UR E S.

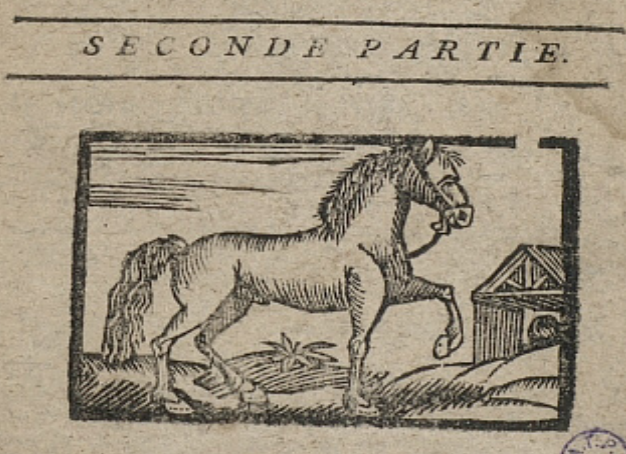

A ROU E N, (ais)

Chez LECRÊNE-LABBEY, rüe dela Groffe-Forloge; N. ${ }^{\circ}$. 2 .

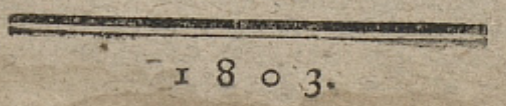

Le Maréchal expert. Seconde partie, A Roven, chez Lecrêne-Labbey, 1803 